\title{
Fractional Calculus Control of Road Vehicle Lateral Stability after a Tire Blowout
}

\author{
Bing Lin LI*, Li ZENG** \\ *Nanjing Forestry University, Nanjing 210037, Jiangsu, China, E-mail: lblqx@njfu.edu.cn \\ **Yangzhou University, Yangzhou 225009, Jiangsu, China, E-mail: lzeng@yzu.edu.cn \\ crossref http://dx.doi.org/10.5755/j02.mech.28524
}

\section{Introduction}

It is a dangerous accident that a vehicle has a tire blowout on a highway. When it occurs, the vehicle's dynamic characteristics, such as, the rolling resistance, the cornering stiffness, and the radial stiffness are changed dramatically, which will easily make the vehicle lose control and cause serious traffic accident, especially on highways. As reported in the National Highway Traffic Safety Administration, about 738 people died due to tire relatedcrashes in 2017 before the vehicle is equipped with the tire pressure monitoring systems (TPMS). Although TPMS can monitor tire pressure, indicating abnormal tire pressure and reducing the occurrence of tire blowout, it has less effect on vehicle control after the tire blowout. This is because that the sudden occurrence of tire blowout causes the driver to be too late to respond. Therefore, it's worth the effort to design an active control method to ensure the vehicle stability after a tire blowout.

For the tire blowout vehicle, active steering control and the braking moment control of the wheels have been studied and developed [1, 2]. Some scholars have emphasized only the design of the control method, such as sliding mode control, LQR optimal control, feed-forward control, to compensate the error between the desired state and actual state after the tire blowout [3 - 5]. Lu Yang proposed a composite stability control strategy for tire blowout electric vehicle with explicit consideration of vertical load redistribution [6]. In order to ensure that the vehicle drive safely after tire blowout until it stops, the vehicle model with tire burst was developed and nonlinear model predictive controller was designed $[7,8]$. Since the instantaneous disturbances due to the tire blowout were difficult to be measured or accurately estimated, the approach of constrained $H_{\infty}$ coordinated control was adopted for the driving stability issues of vehicle [9, 10]. Furthermore, there is also a method to estimate the additional yaw torque after the tire blowout in the light of the vehicle model [11]. However, the mathematical model of the vehicle is difficult to develop accurately, and the problem chattering is prone to occur in the process of control. What's more, the tire-blowout vehicle is non-linear and its parameters are difficult to accurately estimate and predict, thereupon, it is necessary to adopt a robust controller to control the tire-blowout vehicle. Fractional-Order PID Controller (FOPID) can achieve better results than integer-order PID controller, which has been proved to be insensitive to external disturbances [12]; therefore, it is used in this paper.

This paper proposes a controller based on fractional calculus for controlling a tire blowout vehicle. The controller assures the control stability and robustness to the variations in the system parameters and disturbances due to the tire blowout of the vehicle. According to the dynamic characteristics change of the vehicle after the tire burst, the 7 degree of freedom (7DOF) vehicle with a tire blowout is developed. The fractional calculus PID controller is designed based on a 2-DOF vehicle model, which makes the vehicle have ideal handling characteristics, and its output is generally chosen as the control reference value. Because the tire blowout vehicle usually shows under-steer or oversteer, in the paper braking moment is applied to the wheels to enhance the lateral stability and ensure the safety of the vehicle. And then the FOPID is employed to stabilize the tire blowout vehicle.

The organization of the paper is as follows: a vehicle dynamic model with tire blowout is presented in section II, and a fractional calculus PID controller is designed in section III. Two representative simulation results are discussed in section IV. The last section concludes this paper.

\section{Vehicle dynamic modelling with tire blowout}

In order to study the lateral stability of vehicle after a tire blowout, the dynamic characteristic of vehicle should be analyzed. 7DOF model catching the essential features of vehicle is adopted for the control of blowout vehicle [13].

\subsection{Vehicle model}

The 7DOF model include longitudinal and lateral motions, yaw motion and the rotational motions of the four wheels, without considering the factors of air resistance and acceleration resistance, as shown in Fig. 1. The vehicle dynamics equations of motion can be expressed as follows.

Longitudinal motion [14]:

$$
\begin{aligned}
& m\left(\dot{V}_{x}-\gamma \cdot V_{y}\right)=\left(F_{x f l}+F_{x f r}\right) \cos \delta- \\
& -\left(F_{y f l}+F_{y f r}\right) \sin \delta+F_{x r l}+F_{x r r} .
\end{aligned}
$$

The lateral motion:

$$
\begin{aligned}
& m\left(\dot{V}_{y}+\gamma \cdot V_{x}\right)=\left(F_{x f l}+F_{x f r}\right) \sin \delta+ \\
& +\left(F_{y f l}+F_{y f r}\right) \cos \delta+F_{y r l}+F_{y r r} .
\end{aligned}
$$

Yaw motion: 


$$
\begin{aligned}
& I_{z} \cdot \dot{\gamma}=\left[\left(F_{x f l}+F_{x f r}\right) \sin \delta+\left(F_{y f l}+F_{y f r}\right) \cos \delta\right] a+ \\
& +\left[\left(F_{x f r}-F_{x f l}\right) \cos \delta+\left(F_{y f l}-F_{y f r}\right) \sin \delta\right] t_{w 1} / 2+ \\
& +\left(F_{x r r}-F_{x r l}\right) \frac{t_{w 2}}{2}-\left(F_{y r l}+F_{y r r}\right) b,
\end{aligned}
$$

Roll motion:

$$
I_{t w} \cdot \dot{w}_{i}=-R_{w} \cdot F_{x i}-T_{b i}+T_{d i},
$$

where: $\delta$ denotes steering angle at the front wheel; $m$ denotes the vehicle mass; $V_{x}$ and $V_{y}$ denote the longitudinal velocity and the lateral velocity respectively; $\gamma$ denotes the yaw rate; $t_{w 1}$ and $t_{w 2}$ stand for track width of front axle and rear axle respectively; $I_{z}$ denotes the yaw inertia around the $\mathrm{Z}$ axis; $h_{g}$ is the distance from the center of gravity to the ground; $F_{x i}, F_{y i}$ and $F_{z i}$ are the tire longitudinal traction force, the tire lateral traction force and the tire vertical force at the $i$-th wheel respectively ( $i=f l, f r, r l$ and $r r$ denote the left front wheel, the right front wheel, left rear wheels and right rear wheels, respectively); $a$ and $b$ are the distance from front and rear axles to the center of gravity; $l=a+b$ is the distance from front axle to rear axle.

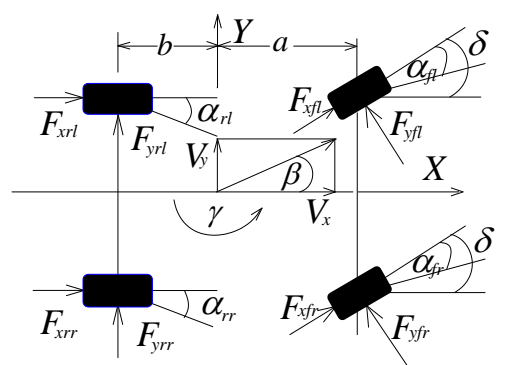

Fig. 1 Vehicle dynamics model with 7DOF

The vertical loads on each wheel are as follows:

$$
\left\{\begin{array}{l}
F_{z_{-} f l}=m g \frac{b}{2 l}-m \dot{V}_{x} \frac{h_{g}}{2 l}-m \dot{V}_{y} \frac{h_{g}}{t_{w 1}} \cdot \frac{b}{l} \\
F_{z_{-} f r}=m g \frac{b}{2 l}-m \dot{V}_{x} \frac{h_{g}}{2 l}+m \dot{V}_{y} \frac{h_{g}}{t_{w 1}} \cdot \frac{b}{l} \\
F_{z_{-} r l}=m g \frac{a}{2 l}+m \dot{V}_{x} \frac{h_{g}}{2 l}-m \dot{V}_{y} \frac{h_{g}}{t_{w 1}} \cdot \frac{a}{l} \\
F_{z_{-} r r}=m g \frac{a}{2 l}+m \dot{V}_{x} \frac{h_{g}}{2 l}+m \dot{V}_{y} \frac{h_{g}}{t_{w 1}} \cdot \frac{a}{l}
\end{array} .\right.
$$

The side angle of each wheel can be calculated as:

$$
\left\{\begin{array}{l}
\alpha_{f l}=\delta-\arctan \left[\left(V_{y}+a \gamma\right) /\left(V_{x}-\frac{t_{w 1}}{2} \gamma\right)\right] \\
\alpha_{f r}=\delta-\arctan \left[\left(V_{y}+a \gamma\right) /\left(V_{x}+\frac{t_{w 1}}{2} \gamma\right)\right] \\
\alpha_{r l}=-\arctan \left[\left(V_{y}-b \gamma\right) /\left(V_{x}-\frac{t_{w 2}}{2} \gamma\right)\right] \\
\alpha_{r r}=-\arctan \left[\left(V_{y}-b \gamma\right) /\left(V_{x}+\frac{t_{w 2}}{2} \gamma\right)\right]
\end{array}\right.
$$

where: $\alpha_{f l}, \alpha_{f r}, \alpha_{r l}, \alpha_{r r}$ denote the side slip angle of each tire.
The longitudinal velocity of each wheel center can be calculated as:

$$
\left\{\begin{array}{l}
V_{t_{-} f l}=\left(V_{x}-t_{w 1} \gamma / 2\right) \cos \delta+\left(V_{y}+a \gamma\right) \sin \delta \\
V_{t_{-} f r}=\left(V_{x}+t_{w 1} \gamma / 2\right) \cos \delta+\left(V_{y}+a \gamma\right) \sin \delta \\
V_{t_{-} r l}=V_{x}-t_{w 2} \gamma / 2 \\
V_{t_{-} r r}=V_{x}+t_{w 2} \gamma / 2
\end{array}\right.
$$

where: $V_{t_{-} f l}, V_{t_{-} f r}, V_{t_{-} r l}$, and $V_{t_{-} r r}$ are the tire longitudinal velocity.

The wheel slip ratio is defined as follows:

$$
\left\{\begin{array}{l}
\eta_{f l}=\left(w_{f l} R-V_{t_{-} f l}\right) / V_{t_{-} f l} \\
\eta_{f r}=\left(w_{f r} R-V_{t_{-} f r}\right) / V_{t_{-} f r} \\
\eta_{r l}=\left(w_{r l} R-V_{t_{-} r l}\right) / V_{t_{-} r l} \\
\eta_{r r}=\left(w_{r r} R-V_{t_{-} r r}\right) / V_{t_{-} r r}
\end{array}\right.
$$

where: $\eta_{f l}, \eta_{f r}, \eta_{r l}, \eta_{r r}$ denote the slip ratio of each wheel and $R$ is the wheel radius.

\subsection{Tire blowout model}

The tire model affects the vehicle's non-linear performance. Dugoff tire model considering the non-linear issue is suitable for the vehicle dynamics simulation ${ }^{16}$. The tire force can be calculated as:

$$
\begin{aligned}
& F_{x}=\frac{C_{x} \eta_{x}}{1-\eta_{x}} f(S), \\
& F_{y}=\frac{C_{y} \tan \alpha}{1-\eta_{x}} f(S),
\end{aligned}
$$

where:

$$
\begin{aligned}
& S=\frac{\mu F_{z}\left(1-\kappa U \sqrt{\eta_{x}^{2}+\tan ^{2} \alpha}\right)\left(1-\eta_{x}\right)}{2 \sqrt{C_{x}^{2} \eta_{x}^{2}+C_{y}^{2} \tan ^{2} \alpha}}, \\
& f(S)= \begin{cases}S(S-2) & S<1 \\
1 & S \geq 1\end{cases}
\end{aligned}
$$

$F_{x}$ and $F_{y}$ are tire longitudinal force and the tire lateral force respectively; $\eta_{x}$ is the longitudinal slip ratio of the wheel; $\alpha$ is the tire lateral angle; $F_{z}$ is the vertical load; $\mu$ is the adhesion coefficient between the tire and the road surface; $\kappa$ is the tire constant; $C_{x}$ and $C_{y}$ are the tire longitudinal stiffness and the tire radial stiffness respectively.

The parameters of longitudinal stiffness, radial stiffness and tire radius are reduced, while the tire-road friction coefficient is increased after tire blowout [9]. Assuming that the change of tire parameters is linear, the changes in properties of the blowout tire are shown in Fig. 2. In the figure, $T 0$ and $T$ are the start time and the end time of the tire burst respectively. Taking the change of the 
longitudinal stiffness of the tire as an example, the longitudinal stiffness function can be expressed by Eq. (13) as:

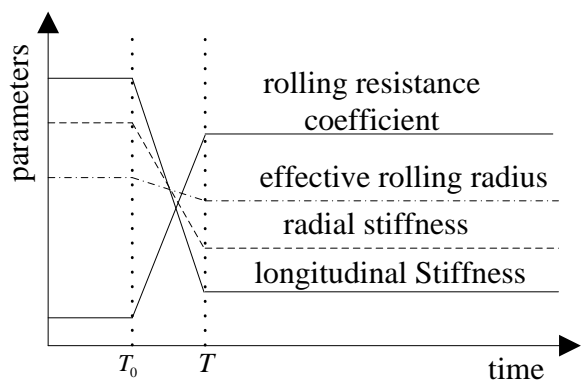

Fig. 2 Changes in properties of the blow out tire

$$
C_{x}^{\prime}=\left\{\begin{array}{lc}
C_{x 0} & \left(0<t<T_{0}\right) \\
\frac{C_{x}^{\prime}-C_{x 0}}{T-T_{0}} \cdot t+C_{x 0} & \left(T_{0}<t<T\right), \\
C_{x}^{\prime} & (t>T)
\end{array}\right.
$$

where: $C_{x 0}$ is the longitudinal stiffness of the tire before the tire blowout; $C_{x}^{\prime}$ is the longitudinal stiffness of the tire after the tire blowout, in this paper, $C_{x}^{\prime}=0.08 C_{x 0}$.

According to formula (13), the parameters of tire cornering stiffness, rolling resistance and effective rolling radius after tire blowout can be obtained. And Dugoff tire blowout model can be acquired by modified these corresponding parameters.

The rolling resistance coefficient of tire on road surface can be described as follow:

$$
f=f_{0}+f_{1}\left(\frac{v_{x}}{100}\right)+f_{4}\left(\frac{v_{x}}{100}\right)^{4},
$$

where: $f_{0}, f_{1}$ and $f_{4}$ denote the tire coefficients, and $f_{0}=$ $0.0085, f_{1}=0.0014, f_{4}=0.0003$.

\subsection{Braking model}

The structure of hydraulic pipeline, proportional valve and wheel cylinder is related to braking pressure of braking system. Moreover, the flow of hydraulic oil in the system is affected by many factors, such as viscosity and density, so its model is very complex and unsuitable for real-time calculation. Therefore, the first and second order empirical models are adopted and shown as follows.

$$
\begin{aligned}
& T_{b}=k_{f} \cdot P, \\
& G(s)=\frac{100}{T B \cdot s+1},
\end{aligned}
$$

where: $T_{b}$ is the braking moment of wheel; $k_{f}$ is brake coefficient and $P$ is brake pneumatic pressure. Eq. (16) represents the transfer function of the first-order braking system with hysteric's characteristics, and $T B$ is the time constant of the system.

The differential form of the first order system can be calculated as:

$$
T \frac{d P}{d t}+P=K P_{i}(t-\tau)
$$

where: $P$ denotes braking pressure; $t$ is time; $K$ is system gain; $P_{i}$ is unit input pressure; $\tau$ is system lag time constant.

\section{Design of controller}

Only by correcting the front wheel steering angle to meet the stability of the vehicle after tire blowout, does it need to rely on the driver's sensitive response. However, the method of differential braking can correct the body attitude of the vehicle no matter it is driving or braking. As we all know, once one of tire is blowout, its pressure drops instantaneously and the rolling resistance on the flat tire increase quickly, which will generate a yaw moment around the $\mathrm{Z}$ axis of the vehicle, and then the yaw moment pulls the vehicle in the direction of the side of the flat tire. In the process of tire blowout, the vehicle shows understeering or over-steering. The way to generate the corrective yaw moment by braking force is chosen in this paper. The relationship between the steering angle of front wheel due to tire blowout, corrective yaw rate response and the braking wheel need to be analyzed first. The distribution of braking moment must be reasonable. Otherwise, the lateral stability cannot be controlled, and the degree of instability will be aggravated and the chance of sideslip and tail flick of the vehicle will be increased. Therefore, according to the front wheel angle and deviation of yaw rate, four cases of vehicle states after a tire burst can be deduced, and the braking wheels are determined, as it shown in Table 1 .

Table 1

Braking moment decision

\begin{tabular}{|c|c|c|c|c|}
\hline $\begin{array}{c}\text { Steer- } \\
\text { ing }\end{array}$ & $\begin{array}{c}\text { Front wheel } \\
\text { steering } \\
\text { angle }\end{array}$ & $\begin{array}{c}\text { Yaw rate } \\
\text { deviation }\end{array}$ & Status vehicle & $\begin{array}{c}\text { Braking } \\
\text { wheel }\end{array}$ \\
\hline Left & $\delta \geq 0$ & $e>0$ & Over steering & Right front \\
\cline { 3 - 5 } & $e \leq 0$ & Under steering & Left rear \\
\hline \multirow{2}{*}{ Right } & $\delta<0$ & $e>0$ & Under steering & Right rear \\
\cline { 3 - 5 } & \multirow{2}{*}{$\delta \leq 0$} & Over steering & Left front \\
\hline
\end{tabular}

\subsection{Reference model}

The linear 2-DOF vehicle model is generally used as reference model in the design of yaw moment controller, which will generate the desired response of the yaw rate and side angle based on steady state condition or approximately first order response. The linear 2-DOF vehicle model is given in the following equation:

$$
\dot{X}=A X+E \delta+B u,
$$

where: $X=\left[\begin{array}{ll}\beta & \gamma\end{array}\right]^{\mathrm{T}}$; yaw moment $u=M$ and front wheel input angle $\delta$ are used in the model;

$$
A=\left[\begin{array}{cc}
-\frac{2\left(C_{f}+C_{r}\right)}{m v} & -1-\frac{2\left(C_{f} l_{f}+C_{r} l_{r}\right)}{m v^{2}} \\
-\frac{2\left(C_{f} l_{f}+C_{r} l_{r}\right)}{J_{z}} & -\frac{2\left(C_{f} l_{f}^{2}+C_{r} l_{f}^{2}\right)}{J_{z} v}
\end{array}\right]
$$


$E=\left[\begin{array}{c}-\frac{2 C_{f}}{m v} \\ -\frac{2 C_{f} l_{f}}{J_{z}}\end{array}\right] ; B=\left[\begin{array}{c}0 \\ \frac{1}{J_{z}}\end{array}\right] ; m$ is mass; $v$ is speed; $C_{f}$ and

$C_{r}$ are lateral stiffness of front and rear wheels respectively; $l_{f}$ and $l_{r}$ are the distance from front axles and rear axles to center of mass respectively; $J_{z}$ is momentum of inertia around vertical axles.

The reference model can be described as:

$$
X_{d}=A_{d} X_{d}+E_{d} \delta
$$

where: $A_{d}=\left[\begin{array}{cc}0 & 0 \\ 0 & -\frac{1}{\tau_{r d}}\end{array}\right] ; E_{d}=\left[\begin{array}{l}0 \\ \frac{k_{r d}}{J_{z}}\end{array}\right]$;

$k_{r d}=\frac{2 C_{f} v}{m v^{2}+2\left(C_{f} l_{f}-C_{r} l_{r}\right)} ; \tau_{r d}=\frac{J_{z} v}{2\left(C_{f} l_{f}^{2}+C_{r} l_{f}^{2}\right)}$.

\subsection{Analysis of control scheme}

The current running state of the vehicle must be obtained before the lateral stability of tire-blowout vehicle is controlled. The corrective yaw moment needs to make the yaw rate and the side angle track its reference value. Because the vehicle stability characteristics are related to the sideslip motion, the side angle is usually bounded to ensure that the vehicle is in a linear region [15]. Once the vehicle has a tire blowout, brake should produce moment to ensure the vehicle running in the linear region. In this paper, the side angle is divided into stable region and unstable region by the method of phase plane. A stability bound defined in [15] is used here, which is formulated as: $B_{1} \beta+B_{2} \beta_{d} \mid \leq 1$. The yaw rate is determined by the method of tolerance zone $\left(|\Delta \gamma|=\left|\gamma-\gamma_{d}\right| \unlhd C \gamma_{d} \mid\right)$, where: $B_{1}=4.386, B_{2}=2.562, C=0.165$ [16]. If the yaw rate and the side angle do not satisfy these two inequalities, the lateral stability control of the vehicle is necessary.

\subsection{Fractional calculus controller}

Fractional calculus extends the order of conventional calculus to arbitrary real numbers. ${ }_{t_{0}} D_{t}^{\varphi} f(t)$ represents basic operators for non-integer order differentiation and integration[17]. It is defined as:

$$
{ }_{t_{0}} D_{t}^{\varphi} f(t)=\left\{\begin{array}{lr}
d^{\varphi} / d t^{\varphi}, \mathfrak{R}(\varphi)>0 \\
1, & \mathfrak{R}(\varphi)=0, \\
\int_{t_{0}}^{t}(d \tau)^{\varphi}, \mathfrak{R}(\varphi)<0
\end{array}\right.
$$

where: $\varphi$ is the order of the differentiation or integration and $t$ and $t_{0}$ are the bounds of operation. The GrünwaldLetnikov (G-L) of the fractional derivative is one of the most commonly definition, which is equivalent under some conditions for a wide class of functions.

According to G-L definition, ${ }_{t_{0}} D_{t}^{\varphi} f(t)$ it can be defined as:

$$
\begin{aligned}
& { }_{t_{0}} D_{t}^{\varphi} f(t)= \\
& =\lim _{h \rightarrow 0} h^{-\varphi} \sum_{j=0}^{\left[\left(t-t_{0}\right) / h\right]}(-1)^{j} \frac{\Gamma(\varphi+1)}{\Gamma(\varphi-j+1) \Gamma(j+1)} f(t-j h),
\end{aligned}
$$

where: $h$ is the calculation step size and $\Gamma(\cdot)$ is the Euler's Gamma function. In numerical calculation, the G-L definition is usually also expresses as:

$$
{ }_{t_{0}} D_{t}^{\varphi} f(t)=h^{-\varphi} \sum_{j=0}^{\left[\left(t-t_{0}\right) / h\right]} b_{j} f(t-j h)
$$

where: $b_{0}=1, \quad b_{j}=\left(1-\frac{1+\varphi}{j}\right) b_{j-1}$.

Since the initial value is zero, the Laplace transformation of fractional calculus operator defined by G-L can be expressed as:

$$
L\left[{ }_{t_{0}} D_{t}^{\varphi} f(t)\right]=s^{\varphi} L[f(t)] .
$$

In Laplace form, fractional order PID controller in parallel structure is given by:

$$
C(s)=k_{p}+\frac{k_{i}}{s^{\lambda}}+k_{d} s^{\varepsilon},
$$

where: $k_{p}, k_{i}$ and $k_{d}$ are the controller gains, and $\lambda$ and $\varepsilon$ are the fractional orders operators. Obviously, for $\lambda=\varepsilon=1$ the controller structure becomes to the conventional PID (proportional-integral-derivative) controller in parallel structure. Compared with conventional PID controller, fractional order PID controller has two more adjustable parameters $\lambda$ and $\varepsilon$, so it could make the control more robust and reasonable. And also the numbers of adjustable parameters are also increased correspondingly, which increase flexibility of system control, so it makes the control output value more close to the expected value easily.

The transfer function corresponds to the time domain fractional differential equation can be expressed as:

$$
u(t)=k_{p} e(t)+k_{i} D_{t}^{-\lambda} e(t)+k_{d} D_{t}^{-\varepsilon} e(t)
$$

where: $D_{t}^{-\lambda} e(t)={ }_{0} D_{t}^{-\lambda} e(t)$.

The discrete time fractional order $\mathrm{PI}^{\lambda} \mathrm{D}^{\varepsilon}$ controller corresponding to the continuous time factional order can be represented as:

$$
\begin{aligned}
& u(k)=k_{p} e(k)+k_{i} h^{\lambda} \sum_{j=0}^{(t / h)} b_{j} e(k-j)+ \\
& +k_{d} h^{-\varepsilon} \sum_{j=0}^{(t / h)} q_{j} e(k-j) .
\end{aligned}
$$

So the equations of the applied moment corresponding to the yaw rate and the side angle are as follows: 


$$
\begin{aligned}
& \Delta M_{\gamma}=k_{p}^{\gamma} e_{\gamma}(k)+k_{i}^{\gamma} h^{\lambda_{\gamma}} \sum_{j=0}^{(t / h)} b_{j} e(k-j)+ \\
& +k_{d}^{\gamma} h^{-\varepsilon_{\gamma}} \sum_{j=0}^{(t / h)} q_{j} e(k-j), \\
& \Delta M_{\beta}=k_{p}^{\beta} e_{\beta}(k)+k_{i}^{\beta} h^{\lambda_{\beta}} \sum_{j=0}^{(t / h)} b_{j} e(k-j)+ \\
& +k_{d}^{\beta} h^{-\varepsilon_{\beta}} \sum_{j=0}^{(t / h)} q_{j} e(k-j),
\end{aligned}
$$

where: $\Delta M_{\gamma}$ and $\Delta M_{\beta}$ are the correction moment of yaw rate and side angle respectively. $k_{p}^{m}, k_{i}^{m}$ and $k_{d}^{m}(m=\gamma, \beta$ denote the yaw rate and side angle, respectively), are the controller gains, and $\lambda$ and $\varepsilon$ are the fractional orders operators.

Because the yaw rate and the side angle are coupled, the distribution coefficient $\eta(0 \leq \eta \leq 1)$ is introduced in the article.

$$
\Delta M=\eta \cdot \Delta M_{\gamma}+(1-\eta) \cdot \Delta M_{\beta}
$$

As for the selection of distributed coefficient, $\eta$ should not only ensure the stability of the vehicle, but also ensure that the vehicle has the ability of trajectory tracking. In the paper, the principle of yaw rate control is adopted when the side angle is small, so $\eta$ is equal to 0.7. Fig. 3 shows the whole structure of FOPID lateral stability control after the tire blowout.

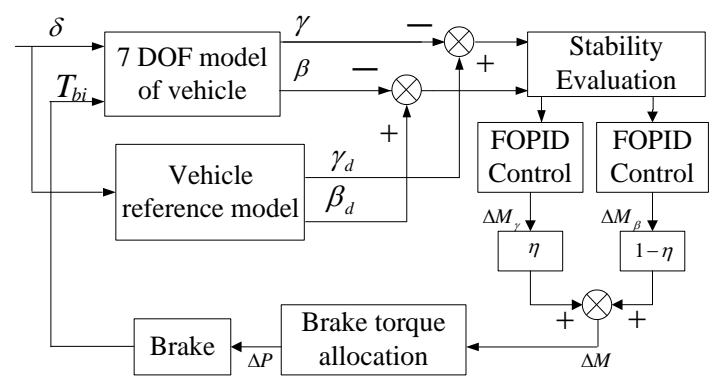

Fig. 3 Block diagram of yaw moment control scheme

\section{Results and discussions}

Computer simulations are performed to verify the

Table 2

Vehicle parameters

\begin{tabular}{|c|c|c|}
\hline Physical parameters & symbol & value \\
\hline $\begin{array}{c}\text { Vehicle total mass, } \mathrm{kg} \\
\text { Distance from vehicle center of gravity } \\
\text { to front axle, m }\end{array}$ & $m$ & 2100 \\
\hline $\begin{array}{c}\text { Distance from vehicle center of gravity } \\
\text { to front axle, m }\end{array}$ & $b$ & 1.256 \\
\hline $\begin{array}{c}\text { Vehicle moment of inertia about yaw } \\
\text { axis Z axis, } \mathrm{kg} \cdot \mathrm{m}^{2}\end{array}$ & $I_{z}$ & 2549 \\
\hline Longitudinal stiffness of tire, $\mathrm{kN} / \mathrm{rad}$ & $C_{x}$ & 60 \\
\hline Cornering stiffness of tire, $\mathrm{kN} / \mathrm{rad}$ & $C_{y}$ & 40 \\
\hline Wheel moment of inertia, $\mathrm{kg} \cdot \mathrm{m}^{2}$ & $J$ & 2.1 \\
\hline Height of vehicle center of gravity, m & $h$ & 0.7 \\
\hline Pavement friction coefficient & $\mu$ & 0.42 \\
\hline Initial speed of vehicle, $\mathrm{km} / \mathrm{h}$ & $V_{0}$ & 96 \\
\hline
\end{tabular}

effectiveness of the control method designed by Simulink after a tire blowout of the vehicle. The experimental vehicle parameters employed for simulation are given in Table 2. The driver did not intervene in the whole running process after a tire blow out.

\subsection{Results and discussion of the vehicle tire blowout in straight driving}

Firstly, supposed the vehicle runs in a straight road. At the beginning, the initial speed of the vehicle is $96 \mathrm{~km} / \mathrm{h}$ and holds the speed for 2 seconds, and then, the left front tire bursts. The failed tire pressure drops instantly and lasts only $0.5 \mathrm{~s}$. Three cases of uncontrolled lateral stability, conventional PID control and fractional order PID (FOPID) control applied to the blowout vehicle are simulated respectively. The simulation results are shown in Figs. 4-8. In Figs. 4-8, the blue solid lines represent the case without control; the red dashed-lines represent the case with PID control, the violet dashed-dotted lines represent the case with FOPID control. It was obvious that, when there was no any control after the tire blowout, the vehicle would deviate from its lane rapidly and it may lead to a traffic accidence. Fortunately, the vehicle stability performance was significantly improved when using the PID controller and FOPID controller, that is to say, the lateral velocity, the side angle and the yaw rate of the tire blowout vehicle have been corrected and numerical returned to 0. Comparing the performance of conventional PID controller with FOPID controller designed in the previous section, it can be seen that the latter one with control

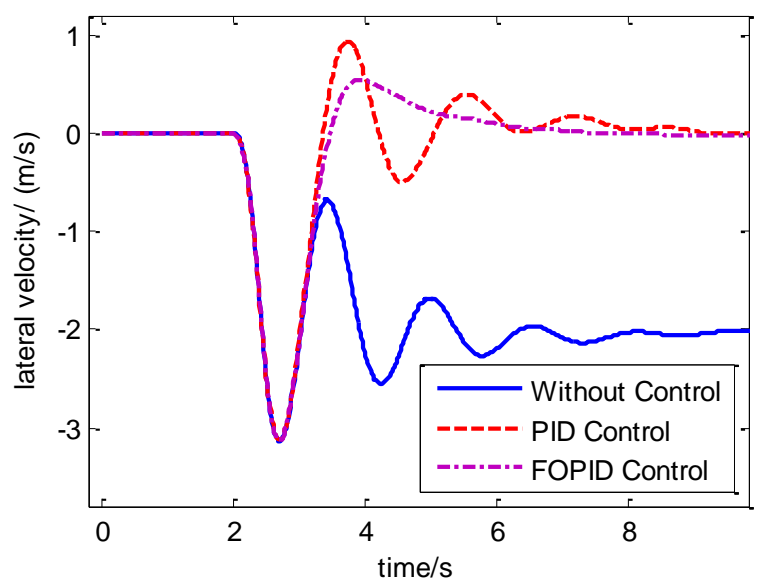

Fig. 4 Lateral velocity

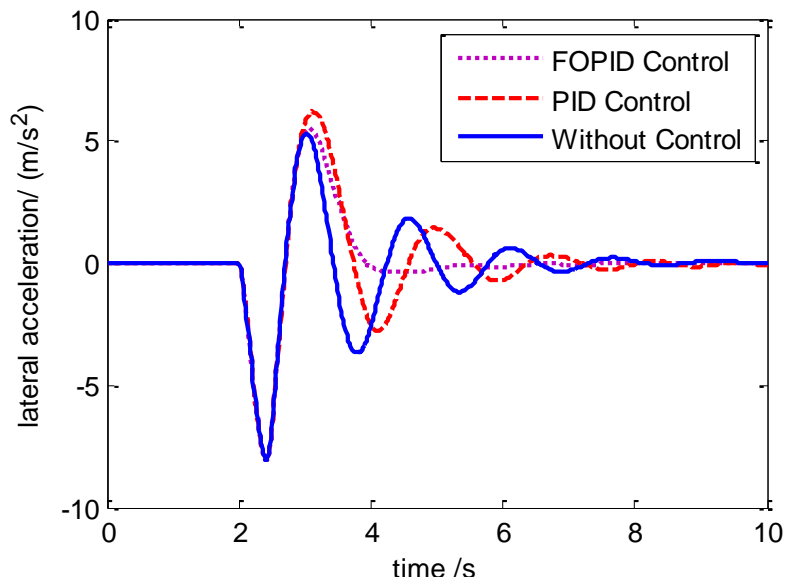

Fig. 5 Lateral acceleration 
parameters $k_{p}=3.95, k_{i}=5, k_{d}=0.5, \varepsilon=-0.59, \lambda=0.49$ has better control performances than that of the former control which has the same value of parameter $k_{p}$ and $k_{d}$. Specifically, the overshoot and the number of oscillations of the latter control less than that of the former. And also the latter's adjustment time shorten, is about 2 seconds, so the vehicle can be restored more quickly.

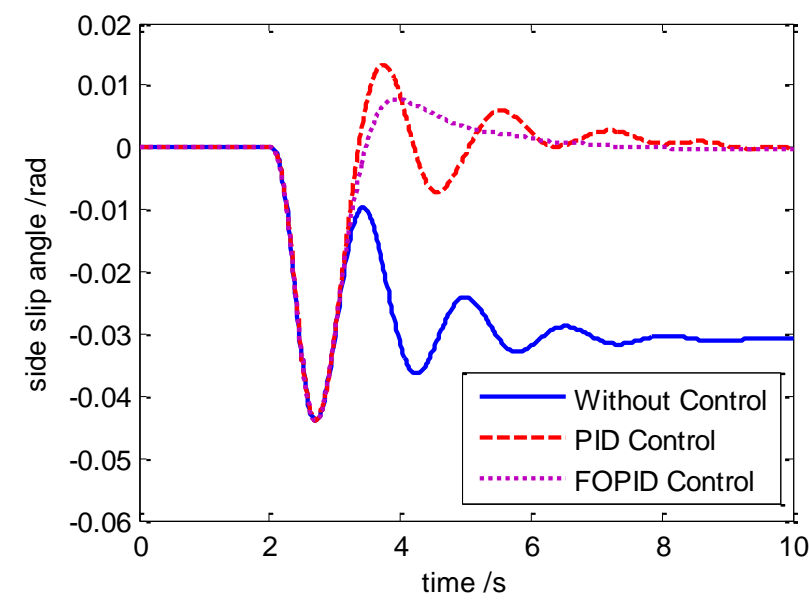

Fig. 6 Variation of vehicle side angle with time

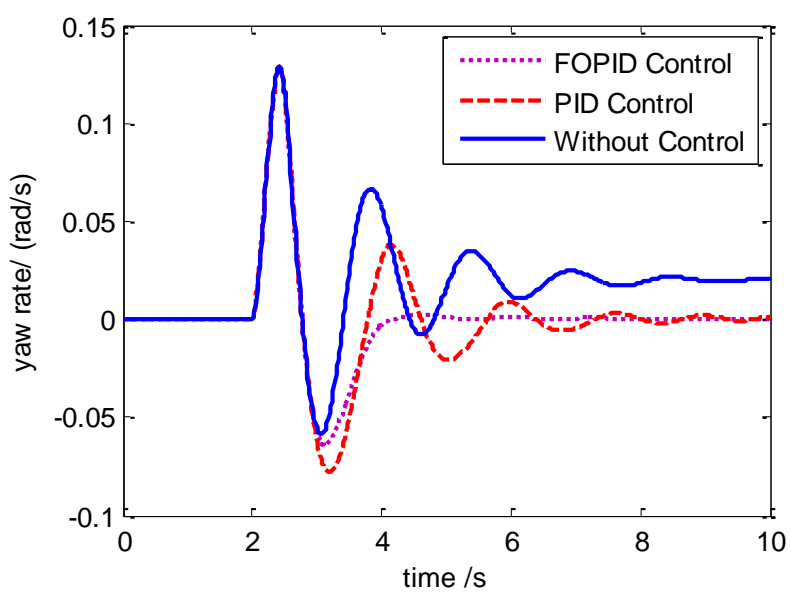

Fig. 7 Yaw rate

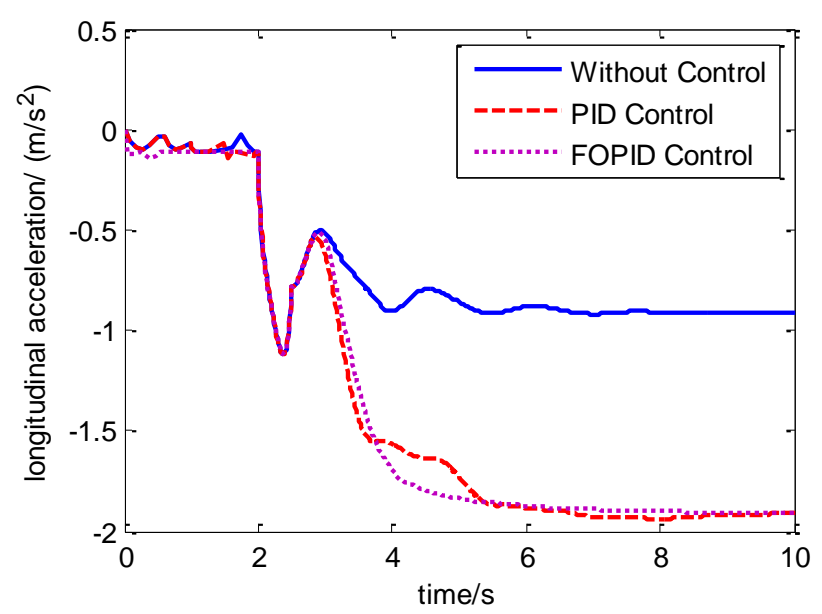

Fig. 8 Longitudinal acceleration

4.2. Results and discussion of the vehicle tire blowout in curve driving

Supposed the vehicle is running on a left-turning road. The vehicle steering wheel angle $\delta=0.012 \mathrm{rad}$, the corresponding radius of the curve's is $218.7 \mathrm{~m}$. The initial speed of the vehicle is also $96 \mathrm{~km} / \mathrm{h}$ and holds the speed for 2 seconds, and then, the left front tire bursts. In this case of tire failure, the simulation results are shown in Figs. 9-12. In Figs. 9-12, the blue solid lines represent the case without control; the red dashed-lines represent the case with PID control, the violet dashed-dotted lines represent the case with FOPID control, the black dotted line represent desired path. When no tire bursts, the vehicle ran in a curve according to the steering angle. Otherwise, once the tire blowout occurred, the vehicle deviated from its own lane and ran to the side of deflated tire if it's not controlled, and the yaw rate and side angle with acute oscillation is undesirable. The vehicle stability performances were also strengthened under the proposed control method. By comparing the control performance of different methods, it can also be known that in the case of FOPID control, with control parameters $k_{p}=-3.95, k_{i}=5, k_{d}=0.5, \varepsilon=-0.59, \lambda=$ $=0.49$ the lateral velocity and yaw rate of the vehicle are closer to the reference value than the PID control results, which can show that the vehicle with blowout is effectively controlled and the stability of the vehicle is guaranteed. In detail, compared with the PID control and FOPID control, the latter has smaller overshoot and fluctuation amplitude in lateral velocity, lateral acceleration, and yaw rate and side angle than the former, which can stabilize the vehicle faster after the tire blowout and improve the safety performance of the vehicle.

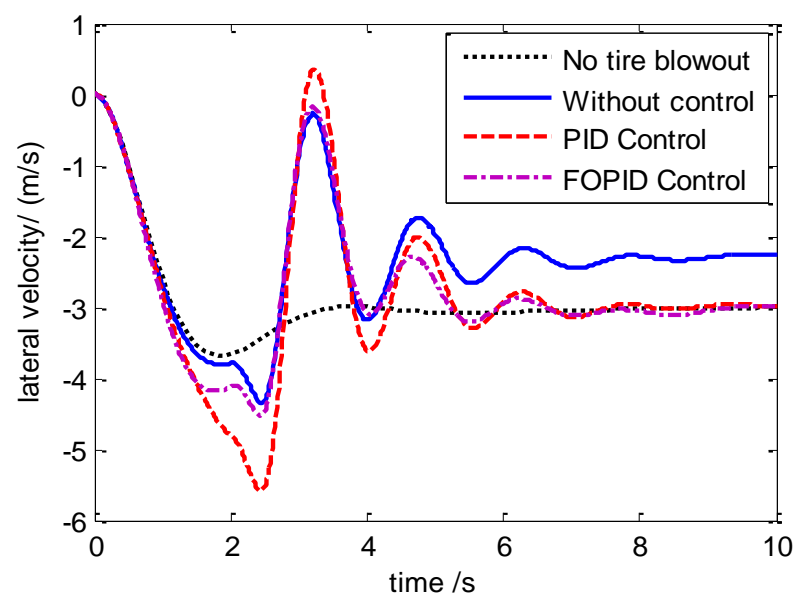

Fig. 9 Lateral velocity

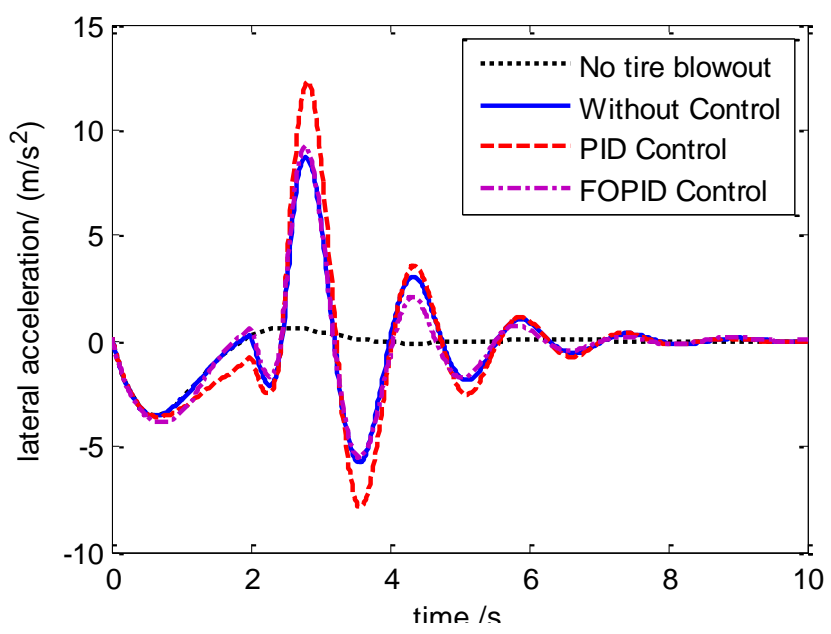

Fig. 10 Lateral acceleration 


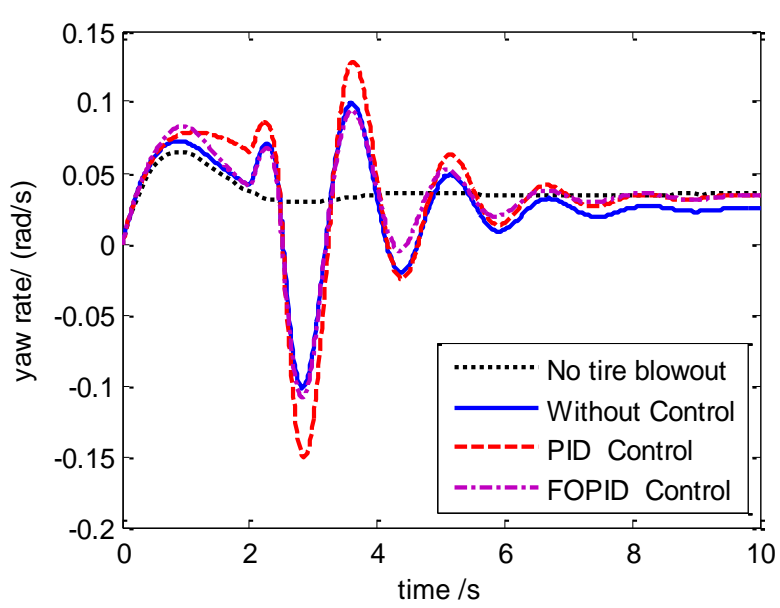

Fig. 11 Yaw rate

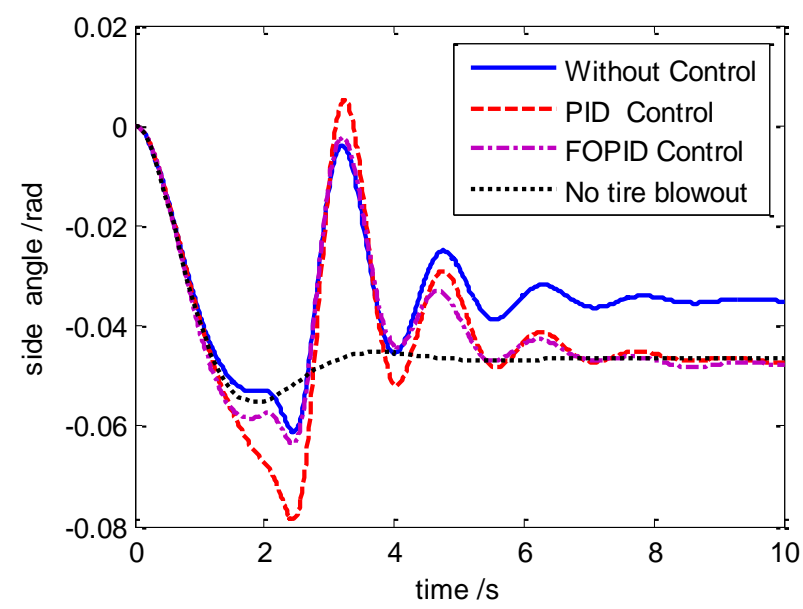

Fig. 12 Vehicle side angle

\section{Conclusions}

A 7-DOF nonlinear vehicle model combining with Dugoff tire blowout model was established. The study looked into the control problem of a vehicle after a tire blowout, and proposed a control method applied to the stability control based fractional order calculus. The method can effectively improve the performance of the vehicle, and has the advantage of robustness. In the process of control, the yaw moment caused by tire blowout is equilibrated by applied the corrective braking force which is decided by the distribution scheme for the vehicle after a tire blowout. The dynamic performance and stability characteristic of the vehicle with the proposed controller are better than the conventional PID controller despite the vehicle runs in straight or turns. This paper only takes the method of braking after the tire blowout, and the steering correction should be researched in the future.

\section{Acknowledgements}

The work was supported by National Natural Science Foundation of China (Grant Nos. 51375427), Natural Science Foundation of the Jiangsu Higher Education Institutions (Grant No. 17KJB150024)

\section{References}

1. Dan, T. H.; Shane, K. L. 2015. Dynamics of tire blowout events and driver assist system for controlling them, Proceedings of the ASME 2015 Dynamic Systems and Control Conference 1: 1-9.

https://doi.org/10.1115/DSCC2015-9621.

2. Wang, F.; Chen, H.; Guo, K. 2017. A novel integrated approach for path following and directional stability control of road vehicles after a tire blow-out, Mechanical Systems and Signal Processing 93: 431-444.

http://dx.doi.org/10.1016/j.ymssp.2017.02.016.

3. Guo, K. H.; Huang, J.; Song, X. L. 2007. Analysis and control of vehicle movement with blown-out tire, Automotive Engineering 29(12): 1041-1045, 1109. (in Chinese).

http://dx.doi.org/10.19562/j.chinasae.qcgc.2007.12.005.

4. Mo, T.; Zhang, X.; Fan, K.2013. Design and simulation of the sliding mode controller for the vehicle blowout process control, International Journal of Vehicle Safety 6(4): 333-346. http://dx.doi.org/10.1504/IJVS.2013.056967.

5. Patwardhan, S.; Tan, S. H. 1997. Experimental results of a tire-burst controller for AHS, Control Engineering Practice 5(11): 1615-1622. http://dx.doi.org/10.1016/S0967-0661(97)10017-X.

6. Yang, L.; Yue, M.; Tian H. Y.; Yao, B. Z. 2020. Tire blow-out control for direct drive electric vehicles using reconfiguration of torque distribution and vertical load, Transactions of the Institute of Measurement and Control 42(8): 1547-1558. http://dx.doi.org/10.1177/0142331219892114.

7. Wang, F.; Chen, H.; Cao, D. 2016. Nonlinear coordinated motion control of road vehicles after a tire blowout, IEEE Transactions on Control Systems Technology 24(3): 956-970. http://dx.doi.org/10.1109/TCST.2015.2472982.

8. Wang, F.; Guo, H. Y.; Chen, H. 2015. Nonlinear model predictive control of blowout tire vehicles and its map-based implementation, Proceedings of the 34th Chinese Control Conference, p. 4043-4048. http://dx.doi.org/10.1109/ChiCC.2015.7260263.

9. Wang, F.; Chen, H.; Guo, H. 2015. Constrained $H \propto$ control for road vehicles after a tire blow-out, Mechatronics 30: 371-382. http://dx.doi.org/10.1016/j.mechatronics.2014.12.007.

10. Jing, H. H,; Liu, Z. Y. 2018. Gain-scheduling robust control for a tire-blow-out road vehicle, Journal of Automobile Engineering 233(2): 344-362. http://dx.doi.org/ 10.1177/0954407017743411.

11. Chen, Q. Li, X.; Zhao, G. 2015. Model of resisting additional yaw torque after tyre blowout on a vehicle, International Journal of Vehicle Safety 8(1): 55. http://dx.doi.org/10.1504/ijvs.2015.066273.

12. Monje, C. A.; Vinagre, B. M.; Batlle, V. F. 2008. Tuning and auto-tuning of fractional order controllers for industry applications, Control Engineering Practice, 16(7): 798-812. http://dx.doi.org/10.1016/j.conengprac.2007.08.006.

13. Jong, H. P.; Woo, S. A. 1999. Yaw-moment control with brakes for improving driving performance and stability, Proceedings of the 1999 IEEE/ASME International Conference on Advanced Intelligent Mechatronics, IEEE Press: 747-752. http://dx.doi.org/10.1109/AIM.1999.803261.

14. Hu, J. J.; Han, Y.; Chen, J. 2017.Integrated control of direct yaw moment control and active suspension system for 4wd vehicles, Mechanika 23(3): 412-424. 
http://dx.doi.org/10.5755/j01.mech.23.3.18481.

15. He, J. J.; Crolla, D. A.; Levesley, M. C.; Manning, W. J. 2006., Coordination of active steering, driveline, and braking for integrated vehicle dynamics control, Proc. Inst. Mech. Eng. D. J. Automob. Eng. 220:14011421.

http://dx.doi.org/10.1243/09544070JAUTO265.

16. Yang, X. J.; Wang, Z. C.; Peng, W. L. 2009. Coordinated control of AFS and DYC for vehicle handling and stability based on optimal guaranteed cost theory, Vehicle System Dynamics 47(1): 57-79. http://dx.doi.org/10.1080/00423110701882264.

17. Podlubny, I. 1999. Fractional-order system and $\mathrm{PI}^{\lambda} \mathrm{D}^{\mu_{-}}$ controller, IEEE Transaction on Automatic Control 44(1): 208-719.

http://dx.doi.org/0018-9286(1999)44:1

$<208$ :FOSASS>2.0.TX;2-I.

\section{B. L. Li, L. Zeng}

\section{FRACTIONAL CALCULUS CONTROL OF ROAD VEHICLE LATERAL STABILITY AFTER A TIRE BLOWOUT}

\section{S u m m a r y}

The lateral stability control of the vehicle can avoid serious traffic accidents when it had a tire blowout during the operation. This article proposes a robust nonlinear control method for controlling vehicle lateral stability after a tire blowout. To be exact, a seven degree of freedom dynamic model of vehicle with modified Dugoff tire model is established. The yaw moment of vehicle is performed by differential braking once the tire blowout occurring. As for control strategy, taking the linear two degree of freedom vehicle model as the reference, using the deviation of yaw rate and the vehicle side angle between the actual value and the reference value as the controller input parameters, the fractional calculus theory is utilized for yaw moment controller which was investigated by regulating the brake moment of blowout vehicle for improving its stability. The results of computer simulation show that the design controller of fractional PID can more effectively enhance the blowout vehicle performance stability compared with the vehicle with the non-control, PID control, no matter in straight road or curve road.

Keywords: safety; blowout vehicle; lateral stability control; fractional calculus; braking moment.

Received February 19, 2021

Accepted December 07, 2021 